\title{
Statistical-mechanical description of classical test-particle dynamics in the presence of an external force field: modelling noise and damping from first principles
}

\author{
I. Kourakis ${ }^{a}$ and A.P. Grecos ${ }^{b}$ \\ a Institut für Theoretische Physik IV, \\ Ruhr-Universität Bochum, D-44780 Bochum, Germany \\ ${ }^{b}$ Euratom - Hellenic Republic Association, \\ University of Thessaly, GR 38334 Volos, Greece
}

(Dated: Submitted 10 October 2005)

\begin{abstract}
Aiming to establish a rigorous link between macroscopic random motion (described e.g. by Langevin-type theories) and microscopic dynamics, we have undertaken a kinetic-theoretical study of the dynamics of a classical test-particle weakly coupled to a large heat-bath in thermal equilibrium. Both subsystems are subject to an external force field. From the (time-non-local) generalized master equation a Fokker-Planck-type equation follows as a "quasi-Markovian" approximation. The kinetic operator thus defined is shown to be ill-defined; in specific, it does not preserve the positivity of the test-particle distribution function $f(\mathbf{x}, \mathbf{v} ; t)$. Adopting an alternative approach, previously proposed for quantum open systems, is proposed to lead to a correct kinetic operator, which yields all the expected properties. A set of explicit expressions for the diffusion and drift coefficients are obtained, allowing for modelling macroscopic diffusion and dynamical friction phenomena, in terms of an external field and intrinsic physical parameters.
\end{abstract}

PACS numbers: 05.20.Dd, 05.10.Gg, 52.25.-b

Keywords: Kinetic theory of random processes, Fokker-Planck equation, test-particle diffusion. 
The link between macroscopic random motion and microscopic particle dynamics has been a long standing fundamental problem, lying in the very heart of Non-Equilibrium Statistical Mechanics. In a generic manner, fluctuations due to particle interactions (collisions) are modelled by a Fokker-Planck-type equation (FPE), typically related to a Langevin-type equation of motion, which may either be derived intuitively, via physical phenomenology or, formally, through kinetic-theoretical arguments [1]. In the latter framework, a number of works in Non-Equilibrium Statistical Mechanics have been devoted to the study of the relaxation of a small subsystem (e.g. a test-particle, t.p.) weakly interacting with a heat bath. A common aim of such studies is the derivation of a kinetic equation, describing the evolution in time of a phase-space probability density function. This is achieved by using either perturbation theory (typically a $B B G K Y$ hierarchy of equations for reduced distribution functions (d.f.) [2, 3]) or formal theories for open systems (e.g. projectionoperator methods 4]). In a generic manner, both approaches rely on a generalized master equation (GME), which is obtained in 2nd order in the (supposedly weak) inter-particle interaction; this is a non-Markovian equation, i.e. is characterized by non-locality in time. The kernel of the GME needs to be evaluated along particle trajectories, so the influence of external force fields on the microscopic laws of motion is in principle expected to modify the form of the collision operator.

This paper is devoted to the rigorous derivation of a Fokker-Planck-type kinetic equation from microscopic dynamics, taking into account the existence of an external force field and interactions (possibly of long range) between particles. This work aims addresses a number of fundamental questions arising in the description of open systems [1, 5] and, as a matter of fact, complements previous studies relying on formal projection operator methods [6, 7, 8], where these issues were first considered with respect to classical systems.

a. The model. We consider a test-particle (t.p.), say $\Sigma$, surrounded by (and weakly coupled to) a homogeneous reservoir $R$; by $\mathbf{X}=(\mathbf{x}, \mathbf{v}) \equiv\left(\mathbf{x}_{\boldsymbol{\Sigma}}(t), \mathbf{v}_{\mathbf{\Sigma}}(t)\right)$ and $\mathbf{X}_{\mathbf{R}} \equiv$ $\left\{\mathbf{X}_{\mathbf{j}}\right\}=\left\{\mathbf{x}_{\mathbf{j}}(t), \mathbf{v}_{\mathbf{j}}(t), j=1,2,3, \ldots, N\right\}$ we shall denote the coordinates of the test- $(\Sigma-)$ and reservoir- $(R-)$ particles, respectively. Both subsystems are subject to an external force field, which affects the particle dynamical trajectories $\{\mathbf{x}(t), \mathbf{v}(t)\}$. In the absence of interactions (collisions), particle trajectories correspond to the solution of the (single particle) dynamical problem of motion $d^{2} \mathbf{x}(t) / d t^{2}=\mathbf{F}_{\mathbf{0}}$. 
The Hamiltonian of the system is:

$$
H=H_{R}+H_{\Sigma}+\lambda H_{I} .
$$

Here $H_{R}\left(H_{\Sigma}\right)$ is the Hamiltonian of the reservoir (t.p.): $H_{R}=\sum_{j=1}^{N} H_{j}+\sum_{j<n} \sum_{n=1}^{N} V_{j n}$. The single-particle Hamiltonian $H_{j}(j=1,2, \ldots, N, \Sigma)$ takes into account the external field. $H_{I}$ stands for the interaction (assumed to be weak: $\lambda \ll 1$ ) between $\Sigma$ and $R$ : $H_{I}=$ $\sum_{n=1}^{N} V_{\Sigma n}$, where $V_{i j} \equiv V\left(\left|\mathbf{x}_{\mathbf{i}}-\mathbf{x}_{\mathbf{j}}\right|\right)(i, j=1,2, \ldots, N, \Sigma)$ is a binary-interaction potential (possibly a long-range e.g. Coulomb-type one). The resulting equations of motion are:

$$
\dot{\mathbf{x}}=\mathbf{v}, \quad \dot{\mathbf{v}}=\mathbf{F}_{\mathbf{0}}(\mathbf{x}, \mathbf{v})+\lambda \mathbf{F}_{\text {int }}\left(\mathbf{x}, \mathbf{v} ; \mathbf{X}_{\mathbf{R}} ; t\right)
$$

The force $\mathbf{F}_{\mathbf{0}}$ is due to the external field. The interaction force $\mathbf{F}_{\text {int }}\left(\mathbf{x}, \mathbf{v} ; \mathbf{X}_{\mathbf{R}} ; t\right)=$ $-\frac{\partial}{\partial \mathbf{x}} \sum V\left(\left|\mathbf{x}-\mathbf{x}_{\mathbf{j}}\right|\right)$ (actually the sum of interactions between $\Sigma$ - and $\mathrm{N} R$-particles) represents a zero-mean Gaussian random process, for a reservoir in a homogeneous equilibrium state [9].

Let us assume that the zeroth-order ('free') problem of motion - namely (2) for $\lambda=0$ yields a known analytical solution in the form: $\mathbf{x}^{(0)}(t)=\mathbf{x}+\int_{0}^{t} d t^{\prime} \mathbf{v}\left(t^{\prime}\right)=\mathbf{M}(t) \mathbf{x}+\mathbf{N}(t) \mathbf{v}$ $\left[\right.$ so $\mathbf{v}^{(0)}(t)=\mathbf{M}^{\prime}(t) \mathbf{x}+\mathbf{N}^{\prime}(t) \mathbf{v}$; the prime denotes differentiation with respect to time $t$, i.e.

$$
\left(\begin{array}{l}
\mathbf{x}^{(0)}(t) \\
\mathbf{v}^{(0)}(t)
\end{array}\right)=\left(\begin{array}{cc}
\mathbf{M}(t) & \mathbf{N}(t) \\
\mathbf{M}^{\prime}(t) & \mathbf{N}^{\prime}(t)
\end{array}\right)\left(\begin{array}{l}
\mathbf{x} \\
\mathbf{v}
\end{array}\right) \equiv \mathbf{E}(t)\left(\begin{array}{l}
\mathbf{x} \\
\mathbf{v}
\end{array}\right) .
$$

with the initial condition $\{\mathbf{x}, \mathbf{v}\} \equiv\left\{\mathbf{x}^{(0)}(0), \mathbf{v}^{(0)}(0)\right\}$, implying $\mathbf{E}(0)=\mathbf{I}$ (the unit matrix). For a given dynamical problem, say in $d$ dimensions $(d=1,2,3)$, the $2 d \times 2 d$ matrix $\mathbf{E}(t)$ in (3) satisfies the group property: $\mathbf{E}(t) \mathbf{E}\left(t^{\prime}\right)=\mathbf{E}\left(t+t^{\prime}\right) \quad \forall t, t^{\prime} \in \Re$, implying $\mathbf{E}(-t)=$ $\mathbf{E}^{-1}(t)$ and a number of explicit relations among the $d \times d$ sub-matrices $\{\mathbf{M}(t), \mathbf{N}(t)\}$, whose form depends on the particular aspects of the dynamical problem into consideration. This working hypothesis requires that a solution of the linearized problem of motion be known. Specific paradigms obeying Eq. (3) include:

(a) linear oscillators, for which (in 1D): $M(t)=N^{\prime}(t)=\cos \omega_{0} t$ and $\omega_{0} N(t)=-\omega_{0}^{-1} M^{\prime}(t)=$ $\sin \omega_{0} t$ (here $\omega_{0}$ is the characteristic spring vibration eigenfrequency);

(b) charged particles (charge $q$, mass $m$ ) subject to a uniform magnetic field $\mathbf{B}=B \hat{z}$ along the $z$-axis, whose (spiral) motion obeys exactly: $\mathbf{M}=\mathbf{I}$ (hence $\mathbf{M}^{\prime}=\mathbf{0}$ ), $\mathbf{N}^{\prime}(t)$ is the rotation matrix: $\mathbf{N}^{\prime}(t)=\left(\begin{array}{ccc}\cos \Omega t & \sin \Omega t & 0 \\ -\sin \Omega t & \cos \Omega t & 0 \\ 0 & 0 & 1\end{array}\right)$ and $\mathbf{N}(t)=\int_{0}^{t} d t^{\prime} \mathbf{R}\left(t^{\prime}\right)$, where $\Omega$ is the 
gyroscopic (cyclotron) frequency $\Omega=q B / m[9$, 10];

(c) the free motion (Chandrasekhar [11] or Rosenbluth-MacDonald-Judd [12]) limit, in the absence of external field(s): $\mathbf{x}(t)=\mathbf{x}+t \mathbf{v}$ [where $\mathbf{v}(t)=\mathbf{v}=$ cst.], viz. $\mathbf{M}=t^{-1} \mathbf{N}=\mathbf{I}$.

b. Statistical formulation. Let $\rho=\rho\left(\left\{\mathbf{X}, \mathbf{X}_{\mathbf{R}}\right\} ; t\right)$ be the total phase-space distribution function (d.f.), normalized to unity: $\int d \mathbf{X} \rho=1$. The equation of continuity in phase space $\Gamma$ reads:

$$
\frac{\partial \rho}{\partial t}+\mathbf{v}_{\mathbf{j}} \frac{\partial \rho}{\partial \mathbf{x}_{\mathbf{j}}}+\frac{\partial}{\partial \mathbf{v}_{\mathbf{j}}}\left(\frac{1}{m} \mathbf{F}_{\mathbf{j}} \rho\right)=0,
$$

where a summation over $j(=1,2,3, \ldots, N, \Sigma)$ is understood.

Defining appropriate ' $s$-body' $(s=1,2,3, \ldots)$ reduced distribution functions $(r d f)$, among which the (1-body-) test-particle $r d f: f(\mathbf{x}, \mathbf{v} ; t)=(I, \rho)_{R} \equiv \int_{\Gamma_{R}} d \mathbf{X}_{\mathbf{R}} \rho$ (normalized to unity), and then integrating the total $((N+1)$-particle) Liouville equation (4), one obtains a $B B G K Y$ hierarchy of coupled evolution equations for the rdfs. This standard procedure 2] has here been adapted to a test-particle problem, namely by defining two types of $r d f$, depending on whether or not the t.p. is included in the particle cluster considered [9]. Truncating the hierarchy, one obtains

$$
\begin{array}{r}
\left(\partial_{t}-L_{0}^{(\Sigma)}\right) f(\mathbf{X} ; t) \approx \lambda^{2} \int d \mathbf{X}_{\mathbf{1}} L_{I} g\left(\mathbf{X}, \mathbf{X}_{\mathbf{1}} ; t\right) \\
\left(\partial_{t}-L_{0}^{(\Sigma)}-L_{0}^{(1)}\right) g\left(\mathbf{X}, \mathbf{X}_{\mathbf{1}} ; t\right) \approx \lambda L_{I} F_{1}\left(\mathbf{X}_{\mathbf{1}}\right) f(\mathbf{X}),
\end{array}
$$

where $L_{0}^{(j)}\left(j \in\left\{\Sigma, 1_{R}\right\}\right)$ is the "free" Liouvillian (in the presence of the field):

$$
L_{0}^{(j)} \cdot=-\mathbf{v}_{\mathbf{j}} \frac{\partial \cdot}{\partial \mathbf{x}_{\mathbf{j}}}-\frac{1}{m_{j}} \frac{\partial}{\partial \mathbf{v}_{\mathbf{j}}}\left(\mathbf{F}_{\mathbf{0}} \cdot\right)
$$

and $L_{I} \equiv L_{\Sigma 1}$ is the binary interaction operator:

$$
L_{I}=-\mathbf{F}_{\mathbf{i n t}}\left(\left|\mathbf{x}-\mathbf{x}_{\mathbf{1}}\right|\right)\left(\frac{1}{m} \frac{\partial}{\partial \mathbf{v}}-\frac{1}{m_{1}} \frac{\partial}{\partial \mathbf{v}_{\mathbf{1}}}\right) .
$$

Recall that the "tags" $\Sigma(1)$ refer to the t.p. (one, any, of the reservoir particles); thus, $f=f(\mathbf{X} ; t), F_{1}\left(\mathbf{X}_{\mathbf{1}_{\mathbf{R}}}\right)$ and $f_{2}\left(\mathbf{X}, \mathbf{X}_{\mathbf{1}} ; t\right)$ denote the $\Sigma$-1-body, $R$-1-body and $\left(1_{R}+\Sigma\right)-2$ body $r d f$ s respectively, while $g=g\left(\mathbf{X}, \mathbf{X}_{\mathbf{1}} ; t\right)$ is the 'two-body' $\left(1_{R}+\Sigma\right)$ correlation function: $g\left(\mathbf{X}, \mathbf{X}_{\mathbf{1}} ; t\right)=f_{2}\left(\mathbf{X}, \mathbf{X}_{\mathbf{1}} ; t\right)-F_{1}\left(\mathbf{X}_{\mathbf{1}}\right) f(\mathbf{X} ; t)$. Note that the mean-field (Vlasov) term $\left(\sim \lambda^{1}\right)$ cancels, for reasons of symmetry, since the reservoir is assumed to be in a homogeneous equilibrium state $F_{1}=n \phi_{e q}\left(\mathbf{v}_{\mathbf{1}}\right)\left(n=N / V\right.$ is the particle density), i.e. $\partial F_{1} / \partial t=L_{0}^{(1)} F_{1}=$ 0 . 
Neglecting correlations at $t=0$, Eqs. (5) lead to the Non-Markovian Generalized Master Equation (GME):

$$
\partial_{t} f-L_{0} f=\lambda^{2} n \int_{0}^{t} d \tau \int d \mathbf{x}_{\mathbf{1}} d \mathbf{v}_{\mathbf{1}} L_{I} U_{0}(\tau) L_{I} \phi_{e q}\left(\mathbf{v}_{\mathbf{1}}\right) f
$$

[here $f=f(\mathbf{x}, \mathbf{v} ; t)]$, where the "free" Liouville operator $L_{0} \equiv L_{0}^{(\Sigma)}$ was defined in (6), $L_{I}$ is the binary interaction Liouville operator $L_{\Sigma 1}$ [cf. (17)] and $U_{0}(\tau)=U_{0}^{(\Sigma)}(\tau) U_{0}^{(1)}(\tau)$ is the evolution operator (propagator) defined by the formal solution of the "free" (collisionless) Liouville equation [i.e. (15a) for $\lambda=0$ ]: $f(t)=e^{L_{0}^{(j)} t} f(0) \equiv U_{0}^{(j)}(t) f(0)$ (for $j \in\{\Sigma, 1\}$ ).

The procedure described here formally amounts to defining the projection: $\mathbb{P} \cdot=$ $\sigma_{R} \int d \Gamma_{R} \cdot$, where $\sigma_{R}$ and $\Gamma_{R}$ denote the reservoir distribution function and phase space, respectively (notice that: $\mathbb{P}^{2}=\mathbb{P}$ ), and seeking an evolution equation for the t.p. rdf $f=\sigma_{R}^{-1} \mathbb{P} \rho$. This may be achieved by defining the complementary projection, say $Q=\mathbb{I}-\mathbb{P}$ (where $\mathbb{I}$ is the identity operator), and then deriving a pair of equations describing the evolution of $P \rho$ and $Q \rho$ in time [6]. This idea has been rigorously elaborated in Refs. [7, 8].

c. A 'quasi-Markovian' approximation - the $\Theta$ - operator. A widely used "markovianization" procedure consists in substituting with the zeroth-order solution, i.e. assuming that $f(t-\tau) \approx e^{-L_{0} \tau} f(t) \equiv U_{0}(-\tau) f(t)$, and then evaluating the kernel asymptotically i.e. taking the time integration limit $t$ to infinity. A kinetic operator is thus formally defined, here denoted as the $\Theta$ - operator. Let us point out that the time-propagator $U(t)$ does not commute with $\Gamma$-space gradients $\frac{\partial}{\partial \mathbf{v}}, \frac{\partial}{\partial \mathbf{x}}$; indeed, one rigorously obtains: $U_{0}^{(j)}(t) \frac{\partial}{\partial \mathbf{v}_{\mathbf{j}}} U_{0}^{(j)}(-t)=\mathbf{N}_{\mathbf{j}}^{\mathbf{T}}(t) \frac{\partial}{\partial \mathbf{x}_{\mathbf{j}}}+\mathbf{N}_{\mathbf{j}}^{\prime T}(t) \frac{\partial}{\partial \mathbf{v}_{\mathbf{j}}}$, for $j=\Sigma, 1_{R}$ (a similar expression holds for $\frac{\partial}{\partial \mathbf{x}_{\mathbf{j}}}$ ) [9]. See that the field inevitably enters - via the dynamical matrices $\mathbf{N}_{j}(t), \ldots-$ the collision term.

Limiting ourselves to a spatially uniform system: $f=f(\mathbf{v} ; t)$ and substituting from (6) and (7) into the GME (8), the 'markovian' approximation described above leads to a parabolic PDE in the form

$$
\frac{\partial f}{\partial t}+\frac{1}{m} \mathbf{F}_{\mathbf{0}} \frac{\partial f}{\partial \mathbf{v}}=\frac{\partial}{\partial \mathbf{v}} \mathbf{A} \frac{\partial f}{\partial \mathbf{v}}+\frac{m}{m_{1}} \frac{\partial}{\partial \mathbf{v}}(\mathbf{a} f),
$$

i.e. a Fokker-Planck-type diffusion equation

$$
\frac{\partial f}{\partial t}+\frac{1}{m} \mathbf{F}_{\mathbf{0}} \frac{\partial f}{\partial \mathbf{v}}=-\frac{\partial}{\partial v_{i}}\left(\mathcal{F}_{i} f\right)+\frac{\partial^{2}}{\partial v_{i} \partial v_{j}}\left(D_{i j} f\right)
$$


(here $\left.i, j=1,2,3 \equiv v_{x}, v_{y}, v_{z}\right)$. Here, $\mathbf{D}=\mathbf{A}$ is a (positive definite) diffusion matrix, which is given by:

$$
\begin{aligned}
\mathbf{A}= & \frac{n}{m^{2}} \int_{0}^{\infty} d \tau \int d \mathbf{x}_{\mathbf{1}} \int d \mathbf{v}_{\mathbf{1}} \phi_{e q}\left(\mathbf{v}_{\mathbf{1}}\right) \\
& \times \mathbf{F}_{\mathbf{i n t}}\left(\left|\mathbf{x}^{(0)}-\mathbf{x}_{\mathbf{1}}{ }^{(0)}\right|\right) \otimes \mathbf{F}_{\mathbf{i n t}}\left(\left|\mathbf{x}^{(0)}(-\tau)-\mathbf{x}_{\mathbf{1}}{ }^{(0)}(-\tau)\right|\right) \mathbf{N}^{\prime T}(\tau) \\
\equiv & \frac{n}{m^{2}} \int_{0}^{\infty} d \tau \mathbf{C}(t, t-\tau) \mathbf{N}^{\prime T}(\tau) .
\end{aligned}
$$

Notice the explicit appearance of the Green-Kubo coefficients (for the interaction forces $\left.\mathbf{F}_{\text {int }}\right)$ : diffusion coefficients are thus related to the force correlation matrix $C_{i j}=$ $\left\langle F_{\text {int }, i}(t) F_{\text {int }, j}(t-\tau)\right\rangle_{R}\left[=C_{i j}(\tau)\right.$, in a stationary process $]$, in agreement with phenomenological stochastic theories. Defining the Fourier transform $\tilde{V}_{k}$ of $V(r)$, this relation takes the form:

$$
D_{r s}^{(V V)}=\frac{n}{m^{2}}(2 \pi)^{d} \int_{0}^{\infty} d \tau \int d \mathbf{v}_{\mathbf{1}} \phi_{e q}\left(\mathbf{v}_{\mathbf{1}}\right) \int d \mathbf{k} e^{i \mathbf{k} \Delta \mathbf{r}} k_{r} k_{m} \tilde{V}_{k}^{2} N^{\prime}{ }_{s m}(\tau)
$$

$\left(d=3\right.$, in a 3D problem), where the exponent $\Delta \mathbf{r}=\mathbf{r}(t)-\mathbf{r}(t-\tau)\left(\right.$ viz. $\left.\mathbf{r}=\mathbf{x}^{(0)}-\mathbf{x}_{\mathbf{1}}{ }^{(0)}\right)$ can be computed by making use of (3); e.g. $\Delta \mathbf{r}(\tau)=\mathbf{N}(\tau)\left(\mathbf{v}^{(0)}-\mathbf{v}_{\mathbf{1}}^{(0)}\right)$, as in paradigms (b) and (c) above, where $\mathbf{M}=\mathbf{I}[9]$. The vector $F$ in the right-hand-side $(r h s): \mathcal{F}_{i}=\mathcal{F}_{i}^{(V)} \equiv$ $\left(1+\frac{m}{m_{1}}\right) \frac{\partial D_{i j}}{\partial v_{j}} \equiv \frac{\partial D_{i j}}{\partial v_{j}}-\frac{m}{m_{1}} a_{i}$, represents the dynamical friction force suffered by the particle, due to interactions with its environment (the reservoir).

Summarizing so far, we have derived a Fokker-Planck kinetic equation, involving an analytical (differential) collision operator; a set of exact expressions for the diffusion coefficients and the dynamical friction vector are thus obtained, to be computed for a given problem (taking into account the external field) either analytically or numerically; cf. e.g. in Ref. [9] for case (b) above.

In the general case of an inhomogeneous d.f. $f=f(\mathbf{x}, \mathbf{v} ; t)$, one obtains the kinetic equation

$$
\frac{\partial f}{\partial t}+\mathbf{v} \frac{\partial f}{\partial \mathbf{x}}+\frac{1}{m} \mathbf{F}_{\mathbf{0}} \frac{\partial f}{\partial \mathbf{v}}=\frac{\partial}{\partial \mathbf{v}} \mathbf{D} \frac{\partial f}{\partial \mathbf{v}}+\frac{\partial}{\partial \mathbf{v}} \mathbf{G} \frac{\partial f}{\partial \mathbf{x}}+\frac{m}{m_{1}} \frac{\partial}{\partial \mathbf{v}}(\mathbf{a} f) \equiv \Theta_{2} f,
$$

where the above definitions still hold; $\mathbf{G}$ is obtained from $r h s(11)$ upon $\mathbf{N}^{\prime T} \rightarrow \mathbf{N}^{T}$. The rhs(13) may readily be cast in the form of a Fokker-Planck operator [i.e. formally as in $r h s$ (10), yet now for 6 variables (in a $3 \mathrm{D}$ problem): $i, j=1,2, \ldots, 6 \equiv x, y, z, v_{x}, v_{y}, v_{z}$. This $6+1$ variable $\mathrm{FPE}$ now involves a $6 \mathrm{D}$ friction vector: $\mathcal{F}=\left(0,0,0 ; \mathcal{F}_{x}^{(V)}, \mathcal{F}_{y}^{(V)}, \mathcal{F}_{z}^{(V)}\right)$ and a $6 \times 6$ diffusion matrix, in the form: $\mathcal{D}^{(\Theta)}=\left(\begin{array}{cc}\mathbf{0} & \frac{1}{2} \mathbf{G}^{T} \\ \frac{1}{2} \mathbf{G} & \mathbf{A}\end{array}\right)$. 
Here is a crucial remark to be made. From a fundamental point of view, the action of a kinetic evolution operator has to preserve the reality, the norm and the positivity of the d.f. $f$ (which is defined e.g. as a normalized probability distribution function); in formal words, a semi-group should be defined. Furthermore, an H-theorem should be satisfied 2], dictating monotonic (irreversible) convergence towards a state of equilibrium (where an entropy function $S \equiv-H$ attains its maximum). In fact, the diffusion matrix $\mathcal{D}^{(\Theta)}$, involved in the $\Theta$-operator, is not positive definite, as can be readily checked (see that $\operatorname{Det} \mathcal{D}^{(\Theta)}=0$ ); therefore (10) determines an ill-defined kinetic operator, whose action does not preserve the positivity of the d.f. $f$ (and neither satisfies an $\mathrm{H}$-theorem). This issue has often been overlooked in the past, since most studies were limited to spatially homogeneous systems (where the problem is not manifested, since $\mathbf{D}^{(V V)}=\mathbf{A} i s$, in principle, positive defininite).

d. Towards a Markovian FPE-the $\Phi$-operator. The forementioned problem has been known in quantum mechanics [4, 5, 13], despite its being overlooked in the classical context [7, 8]. In his remarkable work on quantum open systems [13], E.B. Davies proposed a new Markovian evolution operator, which he formally showed to define a semigroup, and was thus psoposed as a correct description of open systems' evolution towards the equilibrium state of a thermostat. "Davies' device" 4] was recently successfully applied in a (classical) plasma kinetic-theoretical context [9, 10]. The main stepstones and outcome of this method will be briefly exposed in the following.

The alternative $(\Phi-)$ operator introduced by Davies (also see in [7]), essentially amounts to

$$
\Phi \cdot=\lim _{T \rightarrow \infty} \frac{1}{2 T} \int_{-T}^{T} d t^{\prime} U^{(0)}\left(t^{\prime}\right) \cdot U^{(0)}\left(-t^{\prime}\right)
$$

(applied to the kernel of the master equation derived above); recall that $U^{(0)}(t) \equiv \exp L^{(0)} t$ is the zeroth-order (collisionless) evolution operator (propagator).

The analytical construction of the $\Phi$ operator for a given physical problem assumes knowledge of the exact form of the dynamical matrices (defined above), in addition to the reservoir equilibrium state $\phi_{e q}\left(\mathbf{v}_{\mathbf{1}}\right)$ (i.e. typically a Maxwellian). The computation may be quite tedious, yet quite straightforward: it is, in fact, rather simplified in the case of oscillatory test-particle (zeroth-order) motion, as in paradigms (a) and (b) above [unlike in free motion (c), which is unbounded and characterized by a continuum spectrum, giving rise to ill-defined integrals e.g. $\left.\lim _{T \rightarrow \infty} \frac{1}{2 T} \int_{-T}^{T} t^{2} d t\right]$; further details, here omitted for brevity, can be found in $[9]$. 
We may briefly state the outcome of the calculations in two typical cases, namely paradigms (a) and (b) above, where the $\Phi$ operator was shown to provide a well-defined FPE, which was proven to fulfill all the necessary requirements. In both cases, the FP-type kinetic equation obtained features a novel collision term, which accounts for diffusion in real, position space, in addition to velocity space diffusion [present, alone, in the $\Theta-\mathrm{FPE}$ (10)]. Furthermore, a modified cross-velocity-position diffusion part is obtained [replacing the "evil" second term in $r h s(\underline{13})$ ]; finally, the dynamical friction vector $\mathcal{F}$ is modified [cf. the last terms in the two Eqs. below, which are absent in $r h s$ (13)]

In the case of charged particle motion in a uniform magnetic field, the long calculation yields a kinetic equation in the form

$$
\begin{aligned}
& \frac{\partial f}{\partial t}+\mathbf{v} \frac{\partial f}{\partial \mathbf{x}}+\frac{q}{m}(\mathbf{v}\times \mathbf{B}) \frac{\partial f}{\partial \mathbf{v}}=\left(\frac{\partial^{2}}{\partial v_{x}^{2}}+\frac{\partial^{2}}{\partial v_{y}^{2}}\right)\left[D_{\perp}(\mathbf{v}) f\right]+\frac{\partial^{2}}{\partial v_{z}^{2}}\left[D_{\|}(\mathbf{v}) f\right] \\
&+2 \Omega^{-1}\left[\frac{\partial^{2}}{\partial v_{x} \partial y}-\frac{\partial^{2}}{\partial v_{y} \partial x}\right]\left[D_{\perp}(\mathbf{v}) f\right]+\Omega^{-2}\left[D_{\perp}^{(X X)}(\mathbf{v})\right]\left(\frac{\partial^{2}}{\partial x^{2}}+\frac{\partial^{2}}{\partial y^{2}}\right) f \\
&-\frac{\partial}{\partial v_{x}}\left[\mathcal{F}_{x}(\mathbf{v}) f\right]-\frac{\partial}{\partial v_{y}}\left[\mathcal{F}_{y}(\mathbf{v}) f\right]-\frac{\partial}{\partial v_{z}}\left[\mathcal{F}_{z}(\mathbf{v}) f\right]+\Omega^{-1} \mathcal{F}_{y}(\mathbf{v}) \frac{\partial}{\partial x} f-\Omega^{-1} \mathcal{F}_{x}(\mathbf{v}) \frac{\partial}{\partial y} f
\end{aligned}
$$

(cf. variable definitions in Sec. (0a); note the cylindrical symmetry in the collision term (rhs), as imposed by the field $(\sim \hat{z})$. Also note the XY-space diffusion term in the second line. The lengthy expressions for all coefficients (involving multiple integrations, which have been calculated analytically to some extent, and then computed numerically) are exposed elsewhere [9, 10, 14]. It is verified that all of the expected mathematical requirements are fulfilled by this new plasma kinetic operator. Interestingly, by adopting as a working hypothesis that $D_{i j}$ and $\mathcal{F}_{i} / v_{i}$ are constant (approximately true for low t.p. velocities), Eq. (15) models a multi-dimensional Ornstein-Uhlenbeck random process [4], which can be solved exactly [15].

Considering a "1D gas" of linear oscillators (in a parabolic potential field $\sim \omega_{0}^{2} x^{2} / 2$ ), we have obtained:

$$
\begin{array}{r}
\frac{\partial f}{\partial t}+v \frac{\partial f}{\partial x}-\omega_{0}^{2} x \frac{\partial f}{\partial v}=\frac{\partial^{2}}{\partial v^{2}}\left[D_{V V}(v) f\right]+\frac{\partial^{2}}{\partial v \partial x}\left[D_{V X}(v) f\right]+\frac{\partial^{2}}{\partial x^{2}}\left[D_{X X}(v) f\right] \\
-\frac{\partial}{\partial v}\left[\mathcal{F}_{V}(v) f\right]-\frac{\partial}{\partial x}\left[\mathcal{F}_{X}(v) f\right] .
\end{array}
$$

All (scalar) coefficients can be explicitly computed (relying on the formulae derived above), yet are omitted here for brevity. The collision term thus defined (by the rhs) can be shown to be well-defined as a kinetic evolution operator. 
e. Conclusion - discussion. Relying on first statistical mechanical principles, we have discussed the fundamental issues involved in the derivation of a Fokker-Planck kinetic equation (FPE), for the description of test-particle dynamics in the presence of an external field. Exact expressions were derived for the diffusion and drift coefficients, which may be exactly computed for a class of physical systems. We have thus exactly recovered the anticipated role of the Green-Kubo (force) coefficients, relating diffusion coefficients to force correlations, in agreement with phenomenological stochastic theories. The collision mechanism is nevertheless an intrinsic part of the formalism here, and is not plainly represented by ad hoc assumptions on the nature of the process. Furthermore, the external force field appears explicitly in both correlation functions and diffusion/drift coefficients appearing in the Fokker-Planck kinetic equation for the test-particle. The results presented here are qualitatively reminiscent of previous formal studies [6, 7, 8], where these issues were first discussed with respect to classical systems.

\section{Acknowledgments}

The authors acknowledge fruitful discussions, during the course of this work, with L. Brenig, B. Weyssow and D. Carati (ULB, Brussels).

The work of I.K. was partially supported by the Deutsche Forschungsgemeinschaft (Bonn, Germany) through the Sonderforschungsbereich (SFB) 591 - Universelles Verhalten Gleichgewichtsferner Plasmen: Heizung, Transport und Strukturbildung.

[1] See the elucidating discussion in: N. G. Van-Kampen, Fluct. Noise Lett. 1, 3 (2001).

[2] R. Balescu, Equilibrium and Non-Equilibrium Statistical Mechanics, Wiley, 1975; ibid, Statistical Dynamics, Imperial College Press (1997).

[3] G. Kaniadakis, Physics Letters A 310, 377 (2003).

[4] N. G. Van-Kampen, Stochastic Processes in Physics and Chemistry, North-Holland, Amster$\operatorname{dam}(1992)$.

[5] N. G. Van-Kampen, Fluctuation and Noise Letters, Vol. 2 (2001) C7.

[6] A.P.Grecos, in Singularities and Dynamical Systems, S. Pnevmatikos (ed.), North-Holland, Amsterdam (1985). 
[7] A.P.Grecos and C.Tzanakis, Physica A 151, 61 (1988); C. Tzanakis, A. P. Grecos, Physica 149A, 232 (1988).

[8] C.Tzanakis, On the kinetic theory of test-particles weakly-coupled to large equilibrium systems, PhD thesis, U.L.B. Université Libre de Bruxelles (1987); C. Tzanakis, A. P. Grecos, Transport Theor. Stat. Phys. 28, 325 (1999) (also see in Refs. therein).

[9] I.Kourakis, Kinetic Theory of Transport Processes in Magnetized Plasmas, PhD thesis, Université Libre de Bruxelles (2002); online at: www.tp4.rub.de/ ioannis/2002DISS01.pdf.

[10] I.Kourakis, Plasma Phys. Control. Fusion 41, 587 (1999).

[11] S. Chandrasekhar, Principles of Stellar Dynamics, University Press, Chicago (1942).

[12] M. N. Rosenbluth, W. M. McDonald \& D. L. Judd, Phys. Rev. 107, 1 (1957).

[13] E.B. Davies, Comm. Math. Physics 39, 91 (1974).

[14] I. Kourakis, D. Carati \& B. Weyssow, Proc. 2000 ICPP (Québec, Canada), 49 (2001); I. Kourakis, Revista Mexicana de Física 49 (supl. 3), 130 (2003).

[15] I. Kourakis and A.P. Grecos, Comm. Nonlin. Sci. Num. Sim. 8, 547 (2003); see Ch. 10 in [9] for lengthy details. 